\title{
A preliminary study on semen collection, its evaluation, and testicular and sperm morphometries in the wild proboscis monkey (Nasalis larvatus)
}

\author{
Zainal Zahari Zainuddin ${ }^{1}$, Mohamed Reza Mohamed Tarmizi ${ }^{1}$, \\ Yap Keng Chee ${ }^{1}$, Alvin Erut ${ }^{1}$, Wan Nor Fitri ${ }^{2}$, Annas Salleh ${ }^{3,4 凶}$ \\ ${ }^{1}$ Borneo Rhino Alliance, Kota Kinabalu, Sabah, Malaysia \\ ${ }^{2}$ Department of Veterinary Clinical Studies, ${ }^{3}$ Department of Veterinary Laboratory Diagnosis, Faculty of Veterinary Medicine, \\ ${ }^{4}$ Laboratory of Sustainable Animal Production and Diversity, Institute of Tropical Agriculture and Food Security, \\ Universiti Putra Malaysia, 43400 Serdang, Selangor, Malaysia \\ annas@upm.edu.my
}

Received: March 24, 2021

Accepted: August 25, 2021

\begin{abstract}
Introduction: The proboscis monkey (Nasalis larvatus) is an endangered species with a declining population. This article describes the first successful attempt at sperm collection and evaluation, and the testicular and sperm morphometries of the wild proboscis monkey in Sabah, Malaysia. Material and Methods: Eight semen collection procedures using electro-ejaculation and digital manipulation were conducted in three wild adult male proboscis monkeys. A total of 21 ejaculates were collected. The testicular biometry was measured with the aid of ultrasonography. Sample evaluation included semen volume and $\mathrm{pH}$ and sperm concentration, viability, and abnormality. The sperm morphometry was undertaken using phase contrast microscopy. Results: The mean $( \pm \mathrm{SD})$ total testicular volume of these animals was $5.77 \mathrm{~cm}^{3}( \pm 1.58)$. Semen collection by electro-ejaculation resulted in an $84 \%$ success rate, while digital manipulation did not result in any ejaculation. Each animal showed different semen characteristics, where the volume was 5-540 $\mu \mathrm{L}, \mathrm{pH} 8-9$, and sperm concentration $0.041-83.00 \times 10^{6} / \mathrm{mL}$. The percentage of abnormal sperm was high at $76.8 \%( \pm 89.60)$, largely due to midpiece abnormality. Normal sperm had a spherical head and long tail with a head:midpiece:tail length ratio of $1: 2: 8$. Conclusion: The social status of these animals may contribute to the generally low quality of the semen. The techniques and data from this study are useful for future conservation and application of assisted reproductive technology in this species.
\end{abstract}

Keywords: proboscis monkey, Nasalis larvatus, semen collection, sperm morphometry, testicular morphometry.

\section{Introduction}

The proboscis monkey (Nasalis larvatus) is a species of non-human primate that is endemic to Borneo and is distributed along lowland rivers, near-shore islands, and coastal mangrove swamps (24). It is currently listed as an endangered species (3) and is showing a decreasing population trend (14), largely due to continuous hunting and habitat destruction (3). A recent estimation of the total population of the species in Sabah is 5,907 (23). Nasalis larvatus is included in the Wildlife Conservation Enactment 1997 passed in Sabah and classified as a Totally Protected Animal in Schedule I, Section I (19).

Previous studies on the proboscis monkey focused on the population, conservation, feeding ecology, and social dynamics of this species $(4,12,15)$. Studies pertaining to its reproduction mainly shed light on the behaviour, breeding ecology, and breeding seasonality (17), and no literature was found on the spermatology of $N$. larvatus. Sperm morphology, motility and concentration are considered the three most important parameters when assessing semen quality. Despite the decreasing proboscis monkey population, there is no study in the field of reproduction and theriogenology, including research on how assisted reproductive technology (ART) might be implemented.

In macaques, numerous studies have been carried out as they are considered a potential animal model for human and other endangered species of primates (29). It is important to evaluate various semen collection methods in wild animal populations as it aids 
determination of the least risk-prone method that could yield good-quality semen. In primates including macaques, commonly used methods were electroejaculation, digital manipulation, and penile vibratory stimulation $(10,21)$.

Basic theriogenology studies of male animals usually comprise the techniques of semen collection, semen evaluation to obtain the baseline value of the collected semen, and description of the morphology of the sperm. Understanding the morphology of sperm could aid in understanding the evolutionary background of a certain species (31). This article is the first to report successful semen collection using electro-ejaculation and to evaluate semen and testes and sperm morphometry of the wild proboscis monkey.

\section{Material and Methods}

Animals. The study was conducted on three wild adult male proboscis monkeys between November 2017 and December 2019. One monkey inhabited Labuk Bay Proboscis Monkey Sanctuary, one Lok Kawi Wildlife Park, and the third Kinabatangan. All the locations are located in the state of Sabah, Malaysia. The selected proboscis monkeys were adults and weighed between $15 \mathrm{~kg}$ and $22 \mathrm{~kg}$ over the study duration, having overall mean body weight $( \pm$ SD) of $19.79 \mathrm{~kg}( \pm 1.96)$. Individually, the mean body weight for animal ID1 was $19.67 \mathrm{~kg}( \pm 0.47)$, for ID2 was $20.00 \mathrm{~kg}( \pm 0.32)$, and for ID3 was $19.83 \mathrm{~kg}( \pm 2.74)$. During the first anaesthesia, the weights of the proboscis monkeys were estimated based on observation. In subsequent anaesthesia, the weights from previous occasions were used as reference. All three proboscis monkeys were from all-male groups. The study was approved by Sabah Wildlife Department.

Anaesthesia and semen collection. The anaesthesia and semen collection were conducted based on a previous description of a protocol used with pig-tailed macaques (Macaca nemestrina) (29). The proboscis monkeys were baited with a small amount of food and intramuscularly administered (26) the anaesthetic in a pelvic limb using a $3 \mathrm{~mL}$ two-chambered dart with a $1.5 \times 20 \mathrm{~mm}$ plain needle and Vario $1 \mathrm{~V} \mathrm{CO}_{2}$ powered blowpipe system (Telinject, Dudenhofen, Germany). The preparations used were $350 \mu \mathrm{g} / \mathrm{kg}$ medetomidine (Medetomidine $40 \mathrm{mg} / \mathrm{mL}$, Kyron Laboratories, Johannesburg, South Africa) and $80 \mathrm{mg} / \mathrm{kg}$ ketamine $\mathrm{HCl}$ (Ilium Ketamil $100 \mathrm{mg} / \mathrm{mL}$, Troy Laboratories, Glendenning, Australia).

Upon induction of anaesthesia, the proboscis monkeys were intubated with an ID 6.0 cuffed endotracheal tube guided by a laryngoscope. Their body weights were measured using electronic scales. Blood oxygen saturation was measured using VE-H1008 Veterinary Pulse Oximeter (EDAN Instruments, Shenzhen, China) with a probe placed on the nares. End tidal $\mathrm{CO}_{2}$ was measured using an EMMA Mainstream capnometer (Masimo, Irvine, CA, USA). The anaesthetic depth was assessed by jaw tone, anal tone, palpebral reflex, pupillary light reflex and response to noxious stimulus. Supplemental doses of ketamine were administered intravenously to maintain anaesthesia if required. Semen was collected from anaesthetised monkeys by electro-ejaculation or digital manipulation of the penis.

Anesthetised proboscis monkeys were subjected to rectal emptying by cleansing enema using water. Once the anaesthetic depth was stable, the penis was placed inside a conical tube and a Seager Model 14 electroejaculator probe $1.5 \mathrm{~cm}$ in size (Dalzell USA Medical Systems, The Plains, VA, USA) was inserted into the rectum. The voltage used was between $2 \mathrm{~V}$ and $7 \mathrm{~V}$, and 10 stimulations were given for each volt. Stimulation was given for 2-3 seconds with 1-second intervals before the next stimulation. Each ejaculation was collected, placed on a rack in a water bath at $37^{\circ} \mathrm{C}$, and liquefied for $30 \mathrm{~min}$.

Testicular assessment. Following the anaesthetic induction, the dimensions of each testis were measured for length (L) and width (W) using an ultrasound. The volume $(\mathrm{V})$ of each testis was calculated using Hansen's formula $\mathrm{V}=\mathrm{L} \times \mathrm{W}^{2} \times 0.524$ (18) and the total volume was determined by combining the volume of the left and right testes (11). After the completion of the procedures, the proboscis monkeys were moved to a holding cage and administered a reversal drug, Atipamezole (Alzane $5 \mathrm{mg} / \mathrm{mL}$, Zoetis, Madison, NJ, USA), intramuscularly $(26,29)$ at a dose of five times the dose of medetomidine. They were allowed to recover undisturbed before release.

Semen evaluation. Following ejaculation and liquefaction of the coagulated semen, evaluation was conducted. Each ejaculation was denoted as n, signifying a single ejaculate. The semen $\mathrm{pH}$ was measured using $\mathrm{pH}$ indicator strips (Whatman, Cytiva, Global Life Science Solutions, Marlborough, MA, USA). The semen motility was assessed using a CX43-32P01 phase contrast microscope (Olympus, Tokyo, Japan) based on semen criteria previously detailed (30). In brief, a volume of $5 \mu \mathrm{L}$ of each ejaculate was placed on a $37^{\circ} \mathrm{C}$ prewarmed glass slide with a coverslip and 200 spermatozoa cells were counted. The general motility percentage, i.e. the percentage of moving spermatozoa cells, was calculated. The progressive sperm percentage was obtained from the observation of the proportion of spermatozoa cells which were swimming forwards. Good quality semen from all ejaculates was pooled and transferred into a $2 \mathrm{~mL}$ vial float on an expanded polystyrene rack in a water bath at $37^{\circ} \mathrm{C}$.

Aliquots of $5 \mu \mathrm{L}$ were used for semen concentration count and morphology. For a sperm count, $2 \mu \mathrm{L}$ of semen was diluted with $38 \mu \mathrm{L}$ of formal saline to give dilution of $20 \times$. A $10 \mu \mathrm{L}$ volume of the mixture was pipetted into haemocytometer chambers and examined under a light microscope at $400 \times$ magnification to determine the sperm concentration. For morphological examination, thin smears on glass slides were prepared from droplets of diluted semen. If the sperm 
concentration was moderate or below, a dilution ratio of $1: 2$ (aliquot of semen:stain) was used (30) and if the sperm concentration was high, a dilution ratio of $1: 3$ (aliquot of semen:stain) was used (30). Two types of stains were utilised: Diff-Quik for observation of abnormalities and eosin-nigrosin for evaluation of live or dead status of spermatozoa. Sperm abnormalities were divided by site and categorised as in the head, midpiece, or tail region. The percentages of normal, abnormal, live, and dead sperm were subsequently calculated.

Sperm morphometry. The morphology of the sperm head was evaluated after staining a thin smear using Diff-Quik staining procedures. Sperm morphology was evaluated with a DME phase contrast microscope (Leica, Wetzlar, Germany) at $400 \times$ magnification. The percentage of abnormalities of the head, midpiece, and tail was assessed by inspecting at least 200 spermatozoa per sample. Morphometric measurements were taken manually using NIS-Elements imaging software (Nikon, Tokyo, Japan) and in accordance with systems proposed by the World Health Organization (28) and a previous report (2). The length, width, perimeter and area of the head and length of the midpiece, tail, and whole spermatozoon were the measured dimensions. From these, the indices of head elongation, ellipticity, roughness, and regularity were derived using the formulae presented in Table 1 . This morphometric inspection was carried out on a total of 200 spermatozoa from various ejaculates collected from the three proboscis monkeys.

Table 1. Variables and formulae used to calculate the sperm morphometric indices of proboscis monkeys

\begin{tabular}{ll}
\hline Parameter & Variable/Formula \\
\hline Head length $(\mu \mathrm{m})$ & $\mathrm{L}$ \\
Head width $(\mu \mathrm{m})$ & $\mathrm{W}$ \\
Head perimeter $(\mu \mathrm{m})$ & $\mathrm{P}$ \\
Head area $\left(\mu \mathrm{m}^{2}\right)$ & $\mathrm{A}$ \\
Head ellipticity & $\mathrm{L} / \mathrm{W}$ \\
Head elongation & $(\mathrm{L}-\mathrm{W}) /(\mathrm{L}+\mathrm{W})$ \\
Head roughness & $4 \pi\left(\mathrm{A} / \mathrm{P}^{2}\right)$ \\
\hline
\end{tabular}

Data analysis. The data of semen evaluation, sperm and testicular morphometry were analysed descriptively for each proboscis monkey. When statistical analysis was possible, individual animals' parameters were analysed using one-way ANOVA. Pearson's correlation test was used to determine the correlation between the various parts of the spermatozoon. The results were presented as mean $( \pm \mathrm{SD})$ and range. All data were analysed using SPSS version 25 (IBM, Armonk, NY, USA).

\section{Results}

Testicular biometry. Testicular size and scrotal circumference during the collection period are shown in Table 2. All three male proboscis monkeys had a firm scrotum. The mean lengths of the left and right testes were $2.04 \mathrm{~cm}( \pm 0.16)$ and $2.24 \mathrm{~cm}( \pm 0.24)$, respectively and the mean widths were $1.58 \mathrm{~cm}( \pm 0.21)$ and $1.55 \mathrm{~cm}( \pm 0.21)$. These dimensions showed the testes to be slightly oval-shaped with a width:length ratio ranging from $1: 1.29$ to $1: 1.45$. The mean volume of the left testes was $2.77 \mathrm{~cm}^{3}$ $( \pm 0.84)$, while that of the right testes was $2.91 \mathrm{~cm}^{3}( \pm 0.90)$. The total volume calculated was $5.77 \mathrm{~cm}^{3}( \pm 1.58)$.

Semen collection. Semen was collected successfully by electro-ejaculation from all three male proboscis monkeys. The overall success of semen collection using this technique was $84 \%$. Digital manipulation was undertaken only twice, which did not result in ejaculation. In proboscis monkey ID2, no semen was ejaculated during the first attempt with electrostimulation on November 18, 2017, while a subsequent attempt on May 23, 2018 resulted in only expulsion of urine. A subsequent attempt on June 20, 2019 obtained only a few dead spermatozoa. These semen samples were discarded and not included in the data analysis. The other two attempts on proboscis monkey ID2 and all attempts on the other two proboscis monkeys ID1 and ID3 were successful and yielded semen samples useable for analysis. Table 3 summarises the semen collection from the proboscis monkeys. The ejaculated semen was either pale white or light creamy yellow in colour.

Semen characteristics. During semen collection, it was noticed that ejaculation usually yielded semen and some amount of coagulum. Proboscis monkey ID1 produced the highest mean volume of semen at $159.44 \mu \mathrm{L}$ $( \pm 162.59)$. This is significantly $(\mathrm{P}<0.05)$ higher than the volumes from the other two proboscis monkeys. The semen $\mathrm{pH}$ values were generally basic, ranging between 8 and 9. Although statistical analysis of sperm counts to elicit any significant differences between the individual monkeys was not possible, the counts were noted to range broadly, from a low of $41,000 / \mu \mathrm{L}$ to a high of $83,000,000 / \mu \mathrm{L}$. Sperm motility ranged between $5 \%$ and $90 \%$, with no statistically significant difference $(\mathrm{P}>0.05)$ between the three proboscis monkeys. For sperm progression, superiority by a statistically significant $(\mathrm{P}<0.05)$ margin was observed in proboscis monkey ID1.

The percentage of normal sperm (Fig. 1a) was low in all proboscis monkeys, ranging between $10.4 \%$ and $23.2 \%$. In general, sperm abnormalities were observed at the head, midpiece, and tail. In the head, abnormalities detected included macrocephaly, microcephaly (Fig. 1b), bicephaly (Fig. 1c), and detachment. Only one type of abnormality was found in the midpiece: it could be bent (Fig. 1d). In the tail, bending (Fig. 1e), coiling (Fig. 1f), tight coiling (Fig. 1g), and duplication (Fig. 1h) were observed. The percentage of abnormal sperm was consistently high. Midpiece bending, which was between $26.1 \%$ and $46.4 \%$ prevalent $($ mean $=35.40 \% \pm 8.71$, median $=34.55 \%$ ), comprised a large part of this percentage. Abnormalities of the head and tail ranged between $0.8 \%$ and $6.4 \%($ mean $=2.75 \% \pm 2.62$, median $=1.90 \%)$ and between $37.7 \%$ and $51 \%$ (mean $=44.70 \% \pm 5.86$, median $=45.05 \%$ ), respectively. A comparison of semen 
quality among the three proboscis monkeys is shown in Table 4.

Semen morphometry. The mean $( \pm$ SD) head width was $4.50( \pm 0.38)$ and the mean head length was $5.53( \pm 0.41)$. The dimensions showed the heads to be nearly spherical with a width:length ratio of $1: 1.23$, a low ellipticity index of $1.12( \pm 0.07)$, and a very low elongation index of $0.05( \pm 0.03)$. The roughness and regularity indices were $0.96( \pm 0.03)$ and $1.01( \pm 0.07)$, respectively. The mean midpiece and tail lengths were $11.62( \pm 2.31)$ and $44.27( \pm 3.60)$, respectively. In general, the proboscis monkey spermatozoon has a long tail compared to the head and midpiece, with a head:midpiece:tail ratio of about $1: 2: 8$. Determination of correlations between a part of the spermatozoon and its total length showed that the head and total length had a weak positive correlation at $\mathrm{R}$ value of 0.2120 . On the other hand, the midpiece and total length revealed a weak negative correlation at $R$ value of -0.2105 . These correlations were not significant $(\mathrm{P}>0.05)$. Between tail length and total length a moderate positive correlation was shown with $\mathrm{R}$ value of 0.7214 and this was significant $(\mathrm{P}<0.05)$. A summary of the semen morphometry is presented in Table 5.

Table 2. Summary of testicular biometry for the three male proboscis monkeys

\begin{tabular}{llllll}
\hline \multirow{2}{*}{ End point } & \multicolumn{2}{c}{ Left } & \multicolumn{2}{c}{ Right } & Total \\
\cline { 2 - 6 } & Mean & Range & Mean & Range & Mean \\
\hline Length $(\mathrm{cm})$ & $2.04( \pm 0.16)$ & $1.86-2.22$ & $2.24( \pm 0.24)$ & $1.94-2.64$ & - \\
Width $(\mathrm{cm})$ & $1.58( \pm 0.21)$ & $1.49-1.96$ & $1.55( \pm 0.21)$ & $1.18-1.84$ & - \\
Volume $\left(\mathrm{cm}^{3}\right)$ & $2.77( \pm 0.84)$ & $1.40-4.47$ & $2.91( \pm 0.90)$ & $1.47-4.19$ & $5.77( \pm 1.58)$ \\
\hline
\end{tabular}

Table 3. Dates of anaesthesia and semen collection, status, and number of ejaculates

\begin{tabular}{clll}
\hline Collection & Proboscis monkey ID1 & Proboscis monkey ID2 & Proboscis monkey ID3 \\
\hline $1^{\text {st }}$ & July 19, 2018 & November 18, 2017 & September 29, 2018 \\
& $\mathrm{n}=5$ & Discarded & \\
$2^{\text {nd }}$ & August 26, 2018 & May 23, 2018 & \\
& $\mathrm{n}=4$ & Discarded & \\
$3^{\text {rd }}$ & & June 20, 2019 \\
& & Discarded \\
$4^{\text {th }}$ & & November 13, 2019 \\
& & $\mathrm{n}=3$ & \\
$5^{\text {th }}$ & & December 3, 2019 \\
& & $\mathrm{n}=2$ \\
\hline
\end{tabular}

$\mathrm{n}$ - number of ejaculates per animal

Table 4. Semen characteristics of the three proboscis monkeys ID1, ID2 and ID3

\begin{tabular}{|c|c|c|c|c|c|}
\hline Characteristic & ID1 $(n=9)$ & ID2 $(n=5)$ & ID3 $(n=7)$ & Mean $( \pm$ SD) & Range \\
\hline Volume $(\mu \mathrm{L})$ & $159.44( \pm 162.59)^{*}$ & $56.00( \pm 40.30)$ & $52.43( \pm 32.92)$ & $99.14( \pm=121.68)$ & $5.00-540.00$ \\
\hline $\mathrm{pH}$ & $8.25( \pm 0.25)$ & $8.50( \pm 0.5)$ & $8.75( \pm 0.25)$ & $8.50( \pm 0.43)$ & $8.00-9.00$ \\
\hline Concentration $\left(\times 10^{6}\right.$ sperm $\left./ \mathrm{mL}\right)$ & $9.35( \pm 7.65)$ & 0.04 & 83.00 & $25.44( \pm 33.89)$ & $0.04-83.00$ \\
\hline Sperm motility (\%) & $56.67( \pm 26.67)$ & $42.00( \pm 9.80)$ & $26.67( \pm 26.09)$ & $44.00( \pm 26.67)$ & $5.00-90.00$ \\
\hline Sperm progression $(\%)$ & $42.22( \pm 29.36)^{*}$ & $8.00( \pm 2.45)$ & $4.17( \pm 4.49)$ & $22.25( \pm 26.90)$ & $0.00-80.00$ \\
\hline Sperm viability $(\%)$ & $65.74( \pm 0.94)$ & 51.90 & 69.10 & $63.12( \pm 6.65)$ & $51.90-69.10$ \\
\hline Normal sperm (\%) & $13.50( \pm 3.10)$ & 18.30 & 23.20 & $17.13( \pm 5.29)$ & $10.40-23.20$ \\
\hline Abnormal sperm (\%) & $86.50( \pm 3.10)$ & 81.70 & 76.80 & $82.88( \pm 5.29)$ & $76.80-89.60$ \\
\hline Total head abnormality (\%) & $0.85( \pm 0.05)$ & 6.40 & 2.90 & $2.75( \pm 2.62)$ & $0.80-6.40$ \\
\hline Macrocephaly (\%) & $0.45( \pm 0.45)$ & 0.00 & 0.00 & $0.23( \pm 0.45)$ & $0.00-0.90$ \\
\hline Microcephaly (\%) & $0.20( \pm 0.20)$ & 0.00 & 2.90 & $0.83( \pm 1.40)$ & $0.00-2.90$ \\
\hline Bicephaly (\%) & $0.20( \pm 0.20)$ & 0.50 & 0.00 & $0.23( \pm 0.26)$ & $0.00-0.50$ \\
\hline Detached head (\%) & $0.00( \pm 0.00)$ & 5.90 & 0.00 & $1.48( \pm 2.95)$ & $0.00-5.90$ \\
\hline Bent midpiece $(\%)$ & $38.95( \pm 7.45)$ & 37.60 & 26.10 & $35.40( \pm 8.71)$ & $26.10-46.40$ \\
\hline Total tail abnormality (\%) & $46.7( \pm 6.08)$ & 37.70 & 47.70 & $44.70( \pm 5.86)$ & $37.70-51.00$ \\
\hline Bent $(\%)$ & $36.65( \pm 1.65)$ & 23.80 & 44.80 & $35.48( \pm 8.78)$ & $23.80-44.80$ \\
\hline Tightly coiled (\%) & $7.05( \pm 0.92)$ & 0.00 & 0.00 & $3.50( \pm 4.11)$ & $0.00-7.70$ \\
\hline Coiled (\%) & $3.00( \pm 2.83)$ & 13.90 & 1.20 & $5.28( \pm 6.04)$ & $1.00-13.90$ \\
\hline Double-tailed (\%) & $0.00( \pm 0.00)$ & 0.00 & 1.70 & $0.43( \pm 0.85)$ & $0.00-1.70$ \\
\hline
\end{tabular}

Results are presented as means unless otherwise indicated-Statistical analysis was performed for the volume, $\mathrm{pH}$, concentration, sperm motility, and sperm progression only. * - statistically significant difference between animals; $\mathrm{n}$ - number of ejaculates per animal

Table 5. Semen morphometry of proboscis monkeys

\begin{tabular}{llcc}
\hline Spermatozoon part & Parameter & Mean $( \pm$ SD) & Range \\
\hline & Length $(\mu \mathrm{m})$ & $5.53( \pm 0.41)$ & $4.68-6.35$ \\
& Width $(\mu \mathrm{m})$ & $4.50( \pm 0.38)$ & $3.86-5.46$ \\
& Perimeter $(\mu \mathrm{m})$ & $15.31( \pm 0.81)$ & $13.63-16.91$ \\
Head & Area $(\mu \mathrm{m})$ & $17.95( \pm 2.05)$ & $12.97-23.26$ \\
& Elongation index & $0.05( \pm 0.03)$ & $0.02-0.11$ \\
& Ellipticity index & $1.12( \pm 0.07)$ & $1.01-1.25$ \\
& Roughness index & $0.96( \pm 0.03)$ & $0.88-0.98$ \\
& Regularity index & $1.01( \pm 0.07)$ & $0.89-1.13$ \\
\hline Midpiece & Length $(\mu \mathrm{m})$ & $11.62( \pm 2.31)$ & $8.50-16.58$ \\
\hline Tail & Length $(\mu \mathrm{m})$ & $44.27( \pm 3.60)$ & $38.03-49.20$ \\
\hline Total length $(\mu \mathrm{m})$ & & $61.42( \pm 2.22)$ & $57.92-66.80$ \\
\hline
\end{tabular}




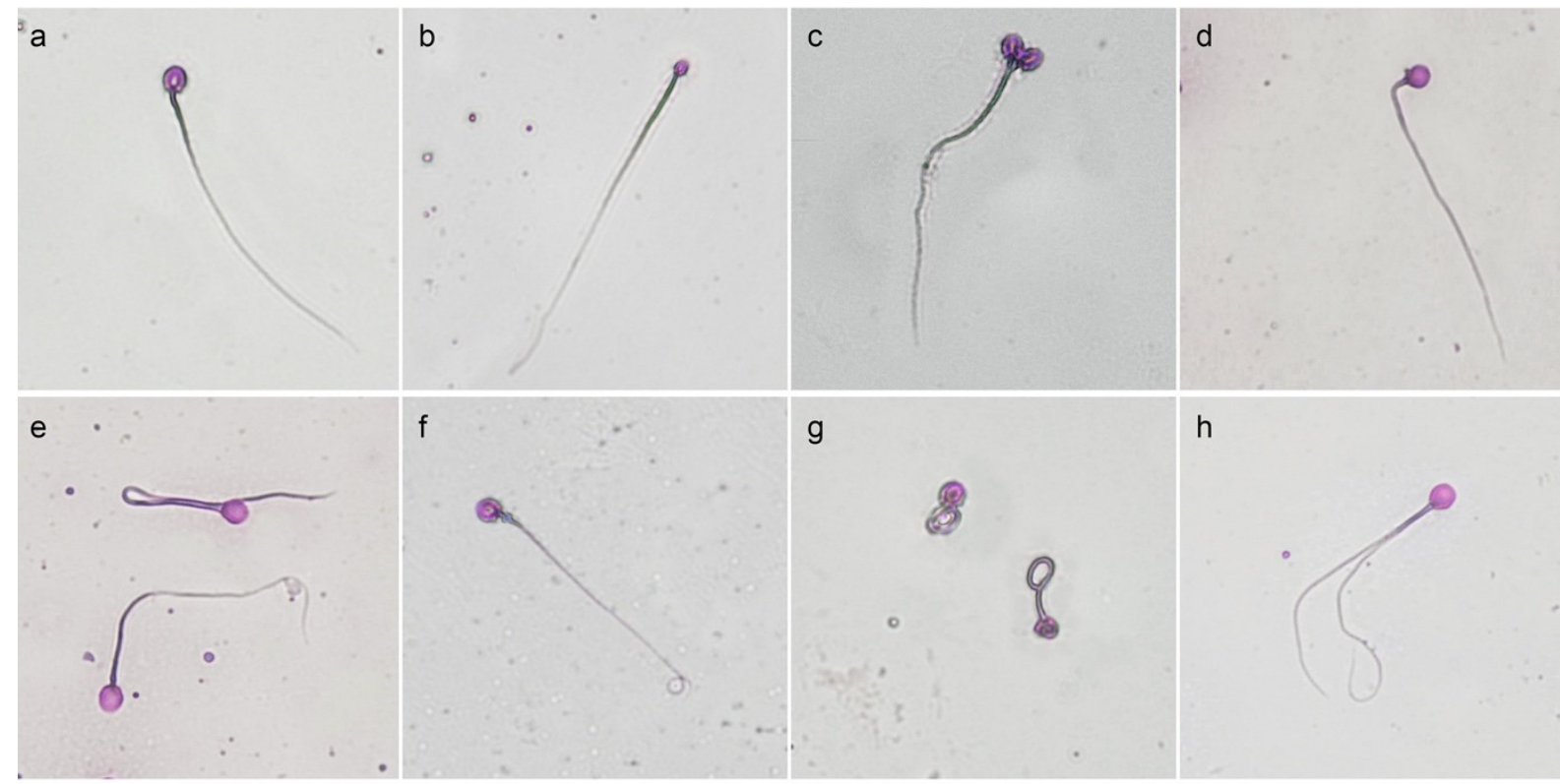

Fig. 1. Morphology of normal and abnormal sperm of proboscis monkeys.

$\mathrm{a}$ - normal sperm; $\mathrm{b}$ - microcephalic; $\mathrm{c}$ - bicephalic; $\mathrm{d}$ - bent midpiece; $\mathrm{e}$ - bent tail; $\mathrm{f}$ - coiled tail; $\mathrm{g}$ - tightly coiled tail; $\mathrm{h}$ - tail duplication

\section{Discussion}

This is the first report to describe a successful semen collection attempt on proboscis monkeys using electro-ejaculation. The high success rate suggests that this technique is reliable and safe to use with the species. The intermediate viability, motility, and concentration and minute volume of the proboscis monkey's sperm indicates low potential for ART to be effective if the technique requires long storage. Sperm motility and viability implied that the semen would not fare well if subjected to cryopreservation. It was found that $>70 \%$ motility is the viability threshold for cryopreservation processes (8). The semen quality necessitates a different ART strategy in this species using semen shortly after obtainment. The most practical and viable option is temporary storage and utilisation of the semen under chilled conditions. A study in primates found artificial insemination with fresh semen to be successful in a rhesus species (6). Nevertheless, with advanced ART such as intracytoplasmic injection (ICSI), storage and cryopreservation of gametes should still be considered despite the low-quality sperm of a given species. This is particularly important to conserve the genetic diversity of valuable, endangered, and small population nonhuman primates such as the proboscis monkey.

After ejaculation, the semen of proboscis monkeys underwent coagulation, which was similarly reported in other primates $(1,29)$. This study showed the semen to be generally low quality with many abnormalities, and semen concentrations to be widely disparate between different individual proboscis monkeys. It should be noted that all of the proboscis monkeys used in this study were from all-male groups. This might have affected the quality of the animals' semen, as social hierarchy and conditions have long been known to affect the sexual behaviour of primates (20). It would be interesting and pertinent to investigate the effect of the presence of females on semen quality. This could be effected by comparing the semen of the studied monkeys to the semen of male proboscis monkeys from mixed-sex groups. The positive effect of female presence on semen quality is known in many mammals $(5,7)$ but was not observed in the tufted capuchin monkey (Sapajus apella) (13). At this point in time, it is impossible to determine whether the presently investigated species of animal naturally has such semen parameters or the findings are related to the anaesthesia, semen collection techniques, and/or influence of their social group composition.

There appeared to be a difference in semen quality between individual proboscis monkeys. However, the current sample size is not adequate to investigate the factors causing the differences in parameters. The factors which could be the causes include the environment, health, social group and hierarchy position, age and feed (20). The effect of the season should be considered in this study since the sampling was conducted over three years in the same individual monkeys. In this study, ID1 would likely be the highestranking male of the three studied based on the observation that it was the largest in its group (25), and this individual had better semen quality in terms of volume and progressive motility. It is desirable to collect semen from this male individual for the purpose of ART. However, the success of semen recovery from other males in this study, which may not have been dominant, promises the wider achievability of gamete recovery in the species. Semen collection from males which may have starkly limited opportunities to mate ensures good genetic diversity. Male proboscis monkeys achieved sexual maturity between 5 and 7 years of age and at about $20 \mathrm{~kg}$ in previous studies $(9,25)$. Successful collection from one small and possibly young or sub- 
adult proboscis monkey is important in indicating that semen collection can be performed earlier, at the time male proboscis monkeys reach a weight of approximately $19 \mathrm{~kg}$. The success of semen collection in this study suggests that males mature earlier than the literature seemed to indicate and that they could be used as donors of genetic material sooner. Modern ART techniques have also allowed the usage of poorly performing sperm for genetic propagation and management. The newest advancements in ART auger well for the proboscis monkey as its population declines, because it is essential to be able to use semen from all male individuals to widen the genetic pool of the species.

In the wild, the breeding season for proboscis monkeys could be affected by the rainfall pattern altering the abundance of leaves which give nutrients, consequently affecting chewing efficiency and rumination (27). It is logical to investigate the environmental impact on the breeding season of the proboscis monkey.

The sperm of the proboscis monkey has an exceptionally long tail compared to the head and the midpiece. The significant correlation between the tail length and the total length of the spermatozoon indicates that a large part of the total length is comprised by the length of the tail. In primates, it was found that the length of the sperm tail indicates high competition between spermatozoa to fertilise an ovum (16).

Concerning sperm abnormalities, it was observed that a preponderance of them were in the midpiece where bends were seen. A previous study of vervet monkeys showed similar findings of a high number of bent midpieces (22).

Some data for semen evaluation were not available in this study, hindering complete statistical analysis between individual proboscis monkeys. This was due to the discarding of poor quality semen because it was of no value for cryopreservation for future conservation efforts. This is the first report of semen collection, semen quality, and basic spermatology in the proboscis monkey. The data and procedures presented serve as baseline parameters of semen quality collected using electro-ejaculation in anaesthetised wild proboscis monkeys and will be useful for the future conservation of the species.

Conflict of Interests Statement: The authors declare that there is no conflict of interests regarding the publication of this article.

Financial Disclosure Statement: The study was funded by the Federal Government of Malaysia and the State Government of Sabah for the conservation effort of wildlife in Sabah.

Animal Rights Statement: Samples and data were collected according to Sabah Wildlife Department (SWD) protocols, under the supervision of SWD employees. Samples and data were collected as part of a routine conservation programme for wildlife in Sabah. Samples and data were non-experimental and regulated by the Wildlife Conservation Enactment 1997. The Malaysian Code of Practice for The Care and Use of Animals for Scientific Purposes does not apply to nonexperimental procedures. Therefore, Animal Experiment Committee approval was not required.

Acknowledgements: The authors acknowledge the staff at Borneo Rhino Alliance and Sabah Wildlife Department and the management of Labuk Bay Proboscis Monkey Sanctuary for their assistance throughout the study.

\section{References}

1. Amboka J.N.O., Mwethera P.G.: Characterization of semen from olive baboons. J Med Primatol 2003, 32, 325-329, doi: 10.1046/j.1600-0684.2003.00039.x.

2. Andraszek K., Banaszewska D., Szeleszczuk O., Niedbała P., Kuchta-Gładysz M.: Comparison of the structure of chinchilla sperm isolated from semen and from the tail of the epididymis. Reprod Domest Anim 2020, 55, 515-522, doi: 10.1111/rda.13646.

3. Boonratana R., Cheyne S.M., Traeholt C., Nijman V., Supriatna J.: Nasalis larvatus. The IUCN Red List of Threatened Species 2020: e.T14352A17945165, doi: 10.2305/IUCN.UK.2020-2.RLTS. T14352A17945165.en.

4. Feilen K.L., Marshall A.J.: Responses to Spatial and Temporal Variation in Food Availability on the Feeding Ecology of Proboscis Monkeys (Nasalis larvatus) in West Kalimantan, Indonesia. Folia Primatol 2020, 91, 399-416, doi: 10.1159/000504362.

5. Fitzpatrick L.J., Gasparini C., Fitzpatrick J.L., Evans J.P.: Male-female relatedness and patterns of male reproductive investment in guppies. Biol Lett 2014, 10, 20140166, doi: 10.1098/rsbl.2014.0166.

6. Gabriel Sánchez-Partida L., Maginnis G., Dominko T., Martinovich C., McVay B., Fanton J., Schatten G.: Live rhesus offspring by artificial insemination using fresh sperm and cryopreserved sperm. Biol Reprod 2000, 63, 1092-1097, doi: 10.1095/biolreprod63.4.1092.

7. Gasparini C., Peretti A.V., Pilastro A.: Female presence influences sperm velocity in the guppy. Biol Lett 2009, 5, 792-794, doi: 10.1098/rsbl.2009.0413.

8. Goericke-Pesch S., Hoffmann B.: Cryopreservation of spermatozoa in veterinary medicine. J Reprod Med Endocrin 2007, 4, 101-105.

9. Hayssen V.D., Van Tienhoven A., Van Tienhoven A.: Asdell's patterns of mammalian reproduction: a compendium of speciesspecific data, Cornell University Press, Ithaca, 2009, doi: 10.7591/9781501734960.

10. Hernández-López L., Cerezo Parra G., Cerda-Molina A.L., Pérez-Bolaños S.C., Díaz Sánchez V., Mondragón-Ceballos R.: Sperm quality differences between the rainy and dry seasons in captive black-handed spider monkeys (Ateles geoffroyi). Am J Primatol 2002, 57, 35-41, doi: 10.1002/ajp.1086.

11. Howard J.G., Brown J.L., Bush M., Wildt D.E.: Teratospermic and normospermic domestic cats: ejaculate traits, pituitarygonadal hormones and improvement of sperm viability and morphology after swim-up processing. J Androl 1990, 11, 204-215.

12. Laman C.J.M., Aziz A.F.: Population estimation of proboscis monkey, Nasalis larvatus with new analysis based on forest types in Sarawak, Malaysian Borneo. J Sustain Sci Manag 2019, 14, 91-101. 
13. Lima J.S., Leão D.L., Oliveira K.G., Brito A.B., Sampaio W.V., Santos R.R., Queiroz H.L., Domingues S.F.: Seminal coagulation and sperm quality in different social contexts in captive tufted capuchin monkeys (Sapajus apella). Am J Primatol 2017, 79, e22643, doi: 10.1002/ajp.22643.

14. Mazlan N., Abd-Rahman M.R., Tingga R.C.T., Abdullah M.T., Anwarali Khan F.A.: Population Genetics Analyses of the Endangered Proboscis Monkey from Malaysian Borneo. Folia Primatol 2019, 90, 139-152, doi: 10.1159/000496022.

15. Meijaard E., Nijman V.: Distribution and conservation of the proboscis monkey (Nasalis larvatus) in Kalimantan, Indonesia. Biol Conserv 2000, 92, 15-24, doi: 10.1016/S00063207(99)00066-X.

16. Møller A.P.: Ejaculate quality, testes size and sperm competition in primates. J Hum Evol 1988, 17, 479-488, doi: 10.1016/00472484(88)90037-1.

17. Murai T.: Mating behaviors of the proboscis monkey (Nasalis larvatus). Am J Primatol 2006, 68, 832-837, doi: 10.1002/ajp.20266

18. Queiroz J.P.A.F., Barros F.F.P.C., Lima G.L., Castelo T.S., Freitas C.I.A., Silva A.R.: Assessment of orchidometry and scrotal circumference in coatis (Nasua nasua). Reprod Domest Anim 2010, 45, e382-e386, doi: 10.1111/j.14390531.2010.01582.x.

19. Legislature of the State of Sabah: Wildlife Conservation Enactment 1997. Sabah 6 1997, Kota Kinabalu, Malaysia.

20. Schaffer N., Cranfield M., Meehan T., Kempske S.: Semen collection and analysis in the conservation of endangered nonhuman primates. Zoo Biol 1989, 8, 47-60, doi: 10.1002/zoo.1430080507.

21. Schneiders A., Sonksen J., Hodges J.K.: Penile vibratory stimulation in the marmoset monkey: a practical alternative to electro-ejaculation, yielding ejaculates of enhanced quality. J Med Primatol 2004, 33, 98-104, doi: 10.1111/j.1600-0684.2004. 00058.x.
22. Seier J.V., van der Horst G., Laubscher R.: Abnormal morphology of vervet monkey sperm. J Med Primatol 1996, 25, 397-403, doi: 10.1111/j.1600-0684.1996.tb00035.x.

23. Sha J.C.M., Bernard H., Nathan S.K.: Status and conservation of proboscis monkeys (Nasalis larvatus) in Sabah, East Malaysia. Primate Conserv 2008, 23, 107-120, doi: 10.1896/052.023.0112.

24. Stark D.J., Nijman V., Lhota S., Robins J.G., Goossens B.: Modeling population viability of local proboscis monkey Nasalis larvatus populations: conservation implications. Endanger Species Res 2012, 16, 31-43, doi: 10.3354/esr00385.

25. Tangah J.: The ecology and behaviour of proboscis monkey (Nasalis larvatus) in mangrove habitat of Labuk Bay, Sabah. PhD dissertation, Universiti Malaysia Sabah, 2012.

26. Theriault B.R., Reed D.A., Niekrasz M.A.: Reversible medetomidine/ketamine anesthesia in captive capuchin monkeys (Cebus apella). J Med Primatol 2008, 37, 74-81, doi: 10.1111/j.1600-0684.2007.00267.x.

27. Thiry V., Clauss M., Stark D.J., Beudels-Jamar R.C., Drubbel R.V., Nathan S.K., Goossens B., Vercauteren M.: Faecal Particle Size in Free-Ranging Proboscis Monkeys, Nasalis larvatus: Variation between Seasons. Folia Primatol 2018, 89, 327-334, doi: 10.1159/000490794.

28. World Health Organization: WHO laboratory manual for the examination of human and sperm-cervical mucus interaction. Cambridge University Press, Cambridge, 1999.

29. Zainuddin Z.Z., Chee Y.K., Mohamed Tarmizi M.R., Ahmad A.H., Payne J.B.: Seminal Evaluation and Cryopreservation of Sperms From The Pig-Tailed Macaque, Macaca nemestrina. J Sustain Sci Manag 2019, 14, 92-99.

30. Zainuddin Z.Z., Mohamed Tarmizi M.R., Chee Y.K., Comizzoli P., Sipangkui S.: First evaluations and cryopreservation of semen samples from Sunda clouded leopards (Neofelis diardi). Animals 2020, 10, 1072, doi: 10.3390/ani10061072.

31. Zhou T., Wang G., Chen M., Zhang M., Guo Y., Yu C., Zhou Z., Si W., Sha J., Guo X.: Comparative analysis of macaque and human sperm proteomes: Insights into sperm competition. Proteomics 2015, 15, 1564-1573, doi: 10.1002/pmic.201400248. 